\title{
Desenvolvimento e caracterização de membranas de quitosana / Cissus Verticil- lata (L.) Nicolson \& C.E. Jarvis
}

\author{
Development and characterization of mem- \\ branes of chitosan / Cissus Verticillata (L.) \\ Nicolson \& C.E. Jarvis
}

\author{
Antônio Alberto Souza Neto ${ }^{1}$, Luanna Abílio Diniz Melquíades de Medeiros ${ }^{1}$, \\ Marcus Vinícius Lia Fook ${ }^{2}$, Rafael Rodrigues de Siqueira ', \\ Rossemberg Cardoso Barbosa ${ }^{2}$, Walter Gleybson Antas de Moraes ${ }^{1}$, \\ Rosana Araújo Rosendo ${ }^{1}$
}

\author{
${ }^{1}$ Unidade Acadêmica de Ciências Biológicas - UACB / UFCG - CEP. 58.708-110, Patos, Paraíba, Brasil. \\ ${ }^{2}$ Laboratório de Avaliação e Desenvolvimento de Biomateriais do Nordeste - CERTBIO/UFCG - CEP: 58.429-900, \\ Campina Grande, Paraíba, Brasil. \\ e-mail: albertosouza0355@gmail.com, luannaabiliod@gmail.com, viniciusliafook@gmail.com, rrodriguesdesiquei- \\ ra@mail.rooseelt.edu,rcbvet@gmail.com,walter_morais18@hotmail.com, cesprodonto@ hotmail.com
}

\begin{abstract}
RESUMO
Os polímeros, devido às suas diversas aplicações e funcionalidades, estão entre os excipientes mais utilizados para a obtenção de formas farmacêuticas, ganhando destaque a quitosana. O objetivo deste trabalho foi desenvolver e avaliar comparativamente membranas de quitosana com diferentes concentrações da espécie vegetal Cissus verticillata (L.) Nicolson \& C.E. Jarvis (insulina), para uso em sistema de liberação de fármaco. Foi realizada coleta, identificação botânica e obtenção do extrato alcoólico bruto (EAB) da planta. Para produção das membranas de quitosana e quitosana com EAB utilizou-se a técnica de evaporação do solvente. As membranas foram divididas nos grupos: MQ (Membrana de quitosana), MQ5 (Membrana de quitosana com 5\% de EAB) e MQ20 (Membrana de quitosana com 20\% de EAB), sendo as amostras caracterizadas pelas técnicas de Microscopia Ótica (MO), Microscopia Eletrônica de Varredura (MEV), de Resistência à Tração e Citotoxicidade. Analisando macroscopicamente as membranas, evidenciou-se mudança de cor à medida que o percentual do EAB foi aumentado, tornando as amostras mais escuras. As análises de MO e MEV evidenciaram membranas com superfície lisa e regular. O ensaio mecânico de resistência à tração revelou diminuição na resistência à tensão quando acrescido 5\% de EAB, e aumento desta com 20\% de EAB. O teste de citotoxicidade mostrou que todas as amostras são viáveis para uso em sistemas biológicos. Logo, conclui-se que foi possível desenvolver membranas de quitosana com diferentes concentrações de EAB, e que o grupo MQ possui uma maior resistência à tensão em relação aos demais grupos.
\end{abstract}

Palavras-chave: Membranas. Quitosana. Plantas medicinais.

\begin{abstract}
The polymers, due to their diverse applications and functionalities, are among the most used excipients to obtain pharmaceutical forms, with emphasis on chitosan. This study aimed to develop and evaluate comparatively membranes of chitosan to different concentrations of the plant species Cissus verticillata (L.) Nicolson \& C.E. Jarvis (insulin), in order to use in a drug delivery system. It was realized data collection, botanical identification and the obtaining of the crude alcohol extract (CAE) of the plant. For the production of chitosan and crude alcohol extracted chitosan membrane, it is used the solvent evaporation technique. The membranes were divided into groups: CM (chitosan membrane), CM5 (5\% of crude alcohol extracted chitosan membrane) e CM20 (20\% of crude alcohol extracted chitosan membrane), being the samples characterized by the techniques of Optical Microscopy (OM), Scanning Electron Microscopy (SEM), of Tensile Strength and Cytotoxicity. Macroscopically analyzing the membranes revealed a change in color as the percentage of CAE increased, making the samples darker. The MO and SEM analyzes showed membranes with smooth and regular surface. The mechanical tensile strength test revealed a decrease in tensile strength when $5 \%$ of CAE was added and increased with $20 \%$ of CAE. The cytotoxicity test showed that all samples are viable for use in biological systems. Therefore, it was concluded that it was possible to develop chitosan
\end{abstract}


membranes with different concentrations of CAE, and that the CM group has a higher resistance to tension in relation to the other groups.

Keywords: Membranes. Chitosan. Medicinal plants.

\section{INTRODUÇÃO}

A utilização de biomateriais na área da saúde é muito comum, sendo estimados cerca de 300 mil nos últimos 10 anos. Essas substâncias podem ser definidas como dispositivos que entram em contato com sistemas biológicos (incluindo fluidos biológicos), com aplicações diagnósticas, vacinais, cirúrgicas ou terapêuticas, dentre outras [1].

Em meio à diversidade dos biomateriais existentes, ganha destaque a quitosana, um polímero natural derivado da quitina. Esta substância tem inúmeras características tais como o fato de ser biodegradável, biocompatível, não tóxico e com atividade antimicrobiana, somando a isso o fato de ter um baixo custo [2].

Nos últimos anos, biomateriais originados da quitosana, são empregados em diversos mecanismos, tais como regeneração tecidual, particularmente para cartilagem, dispositivos de liberação controlada de fármacos e sistemas de imobilização de células em gel [3]. Logo, este polímero tem sido vastamente utilizado na veiculação de medicamentos, bem como na engenharia de tecidos aplicada à odontologia e diversas áreas médicas [4].

Membranas de quitosana estão sendo bastante estudadas por grupos de pesquisa, devido a sua capacidade de acelerar a cicatrização tecidual, uma vez que envolve vários mecanismos como coagulação, síntese e deposição de matriz celular, fibroplasia, dentre outros [5].

$\mathrm{Na}$ literatura, o uso de biomateriais está em crescimento, uma vez que estes apresentam características capazes de controlar a liberação de substâncias como fármacos. Dentre os fármacos, têm-se os fitoterápicos, que são obtidos a partir de derivados vegetais, apresentando inúmeras aplicações na cura das doenças [6].

A Fitoterapia foi a primeira medicina do homem, sendo que as plantas são uma fonte de medicamentos para os seres humanos desde tempos imemoriais. Neste sentido, a terapia através das plantas é a forma mais antiga de cuidados de saúde conhecido pela humanidade [7, 8].

O Brasil é um país de imensa diversificação de espécies vegetais, que muitas vezes são utilizadas como matérias-primas para a fabricação de fitoterápicos que podem ser usados para o tratamento de inúmeras enfermidades, como o diabetes melito [9].

Uma dessas espécies é a Cissus verticillata (L.) Nicolson \& C.E. Jarvis, que pertence à família Vitaceae. Esta planta é conhecida como "Insulina vegetal" existindo, porém, outras nomenclaturas populares menos comuns como cortina japonesa, cipó jucá, anil trepador, uva braba, dentre outros [10].

Tem-se buscado alternativas de administração da insulina de forma menos traumática, contribuindo para minimizar ou mesmo eliminar o incômodo sofrido por inúmeros indivíduos portadores de diabetes que já se encontram naturalmente sensibilizados em virtude das limitações impostas pela doença, sendo o emprego de fitoterápicos uma dessas formas opcionais de terapêutica [11].

Mediante a aprovação da Política Nacional de Práticas Integrativas e Complementares, prevendo o tratamento com plantas medicinais e fitoterápicas no Sistema Único de Saúde - SUS, conforme disposto na Portaria 971 de três de Maio de 2006 do Ministério da Saúde; e da Política Nacional de Plantas Medicinais e Fitoterápicos através do Decreto $\mathrm{n}^{\circ} 5.813$, de 22 de Junho de 2006, este trabalho propôs-se a desenvolver e caracterizar membranas de quitosana com diferentes concentrações de Cissus verticillata (L.) Nicolson \& C.E. Jarvis, a fim de avaliar sua possível utilização em pacientes portadores de diabetes melito tipo 2.

\section{MATERIAIS E MÉTODOS}

\subsection{Materiais}

- $\quad$ Quitosana - de média massa molecular, com grau de desacetilação em torno de $90 \%$ e grau de cristalinidade de aproximadamente 50\%; produzida no CERTBIO (Laboratório de Avaliação e Desenvolvimento de Biomateriais do Nordeste) - UFCG.

- $\quad$ Ácido Acético Glacial P.A., Sigma Aldrich ${ }^{\circledR}$.

- Hidróxido de amônio 28-30\% P.A. - Massa molar 35,05 g/mol, Neon.

- $\quad$ Extrato alcoólico de Cissus verticillata (L.) Nicolson \& C.E. Jarvis. 


\subsection{Metodologia}

\subsubsection{Tipo e Local da Pesquisa}

Foi desenvolvida uma pesquisa laboratorial, in vitro e quantitativa no CERTBIO, localizado na Unidade Acadêmica de Engenharia de Materiais, na Universidade Federal de Campina Grande/ UFCG.

\subsubsection{Coleta e Identificação da Planta}

As folhas de Cissus verticillata (L.) Nicolson \& C.E. Jarvis (Figura 1) foram coletadas no Horto de Plantas Medicinais do Instituto de Pesquisa em Fármacos e Medicamentos (IPeFarM) da Universidade Federal da Paraíba (UFPB), onde a planta é cultivada.

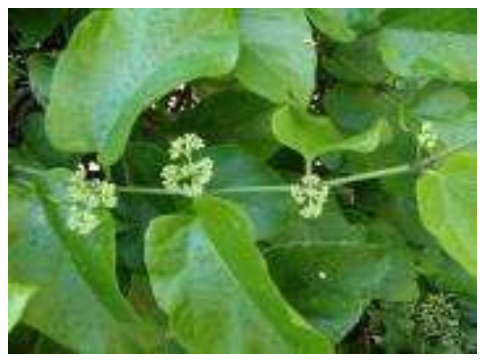

Figura 1: Folhas e frutos de Cissus verticillata (L.) Nicolson \& C.E. Jarvis [12]

Com a finalidade de identificar botanicamente a espécie vegetal utilizada no estudo, partes aéreas (folhas e frutos) de plantas adultas frescas foram prensadas sendo, em seguida, postas para secagem em estufa artesanal a uma temperatura de $38^{\circ} \mathrm{C}$ por 72 horas, para confecção de exsicatas. Amostras representativas dessas exsicatas foram depositadas no Herbário CSTR, localizado no Centro de Saúde e Tecnologia Rural (CSTR/UFCG), sob número de tombo - CSTR 5170.

O extrato alcoólico bruto (EAB) de Cissus verticillata (L.) Nicolson \& C.E. Jarvis empregado foi obtido através do método de remaceração até a extração exaustiva do material vegetal, sendo obtido ao final um material escuro, higroscópico, denominado extrato alcoólico bruto (EAB), com massa de 85,5g (Figura 2).
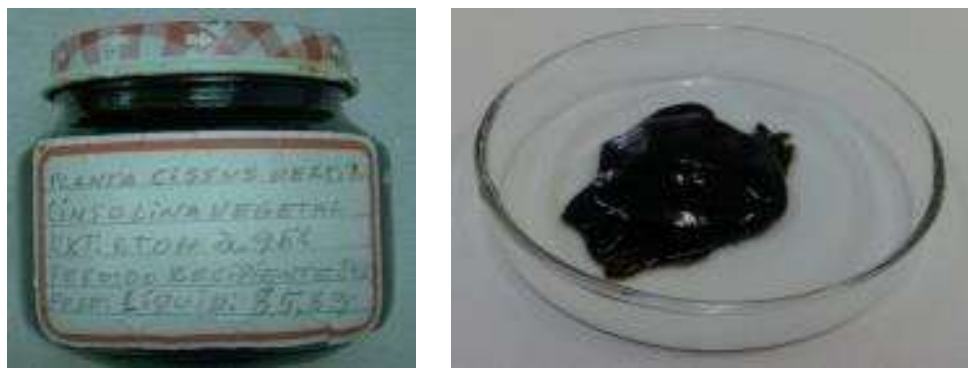

Figura 2: Extrato alcoólico bruto de Cissus verticillata (L.) Nicolson \& C.E. Jarvis [12].

\subsubsection{Metodologias da preparação das membranas de quitosana sem e com a incorporação da droga vegetal}

A solução de quitosana foi preparada pela dissolução de $30 \mathrm{~g}$ de quitosana em 1,5 L de uma solução a $1 \%$ (v/v) de ácido acético glacial sob agitação mecânica durante 24 h a $435 \mathrm{rpm}$ (Figura 3). Foram adicionados $20 \mathrm{~mL}$ da solução em placas de Petri em acrílico com diâmetros de 55x13 mm. 


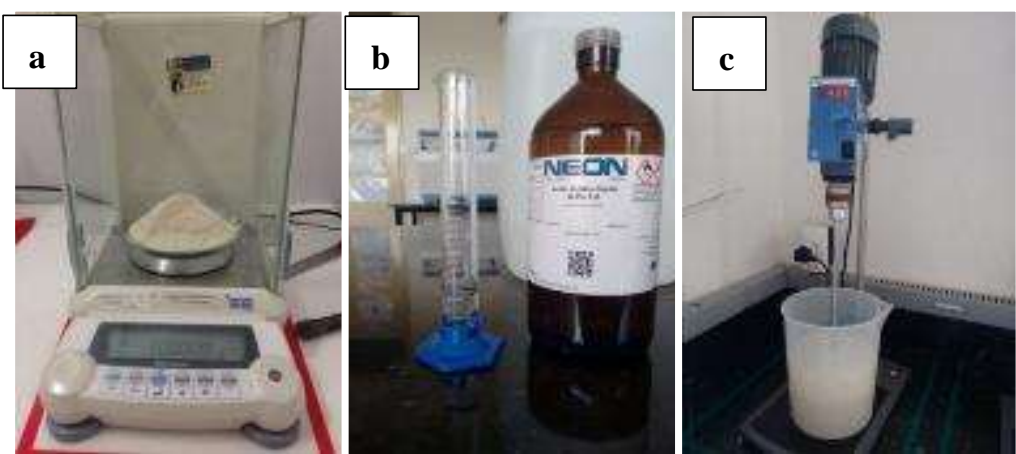

Figura 3: $30 \mathrm{~g}$ de quitosana (a), $15 \mathrm{~mL}$ de ácido acético glacial (b), solução de quitosana com ácido acético sob agitação mecânica (c).

A preparação das membranas contendo a droga vegetal foi realizada dissolvendo-se em $100 \mathrm{~mL}$ da solução de quitosana, $5 \%$ e $20 \%$ da droga vegetal, proporcionalmente à concentração de quitosana empregada. As concentrações da droga vegetal foram selecionadas tomando como base os estudos desenvolvidos por ROSENDO [12] que utilizou 1,0 grama de pó das folhas de Cissus verticillata (L.) em pacientes, sendo essa dosagem considerada ideal para ser empregada pela população como terapêutica.

Essa solução foi colocada também no agitador mecânico IKA RW20 durante $2 \mathrm{~h}$ a $550 \mathrm{rpm}$, até que a droga vegetal dissolvesse de forma homogênea (Figura 4). Da mesma forma, foram vertidos $20 \mathrm{~mL}$ de cada uma das soluções obtidas em placas de Petri em acrílico (Figura 5). As placas foram então acondicionadas em estufa bacteriológica a $35,7^{\circ} \mathrm{C}$ por 7 dias para evaporação do ácido e formação da membrana.
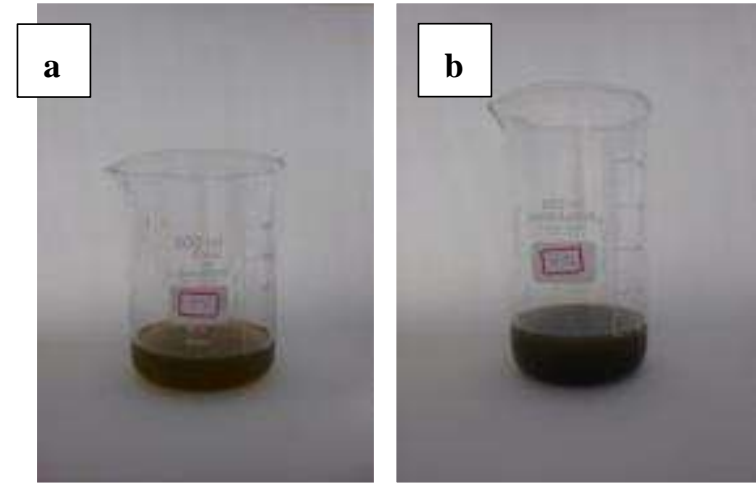

Figura 4: Soluções de quitosana com extrato vegetal a 5\% (a) e 20\% (b) respectivamente após a agitação mecânica.

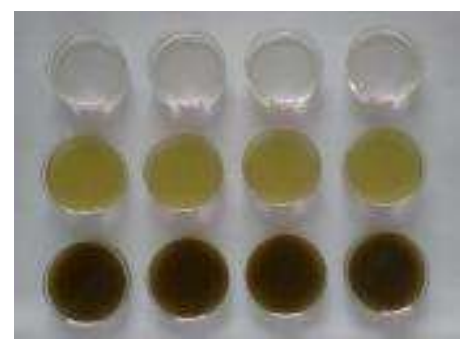

Figura 5: Placas de Petri cada uma contendo $20 \mathrm{~mL}$ de solução, sendo quatro com solução de quitosana pura, quatro com solução de quitosana com $5 \%$ de $\mathrm{EAB}$ e quatro com solução de quitosona com $20 \%$ de $\mathrm{EAB}$.

Após a secagem das membranas de quitosana sem e com a incorporação da droga vegetal, nas concentrações de $5 \%$ e $20 \%$, as mesmas foram retiradas da estufa para a neutralização.

\subsubsection{Processo de Neutralização com Hidróxido de Amônio}

No preparo da solução de hidróxido de amônio, foi dispensado em um balão volumétrico $500 \mathrm{~mL}$ de água destilada, estando a capela para exaustão de gases da SPLabor ligada, e incorporado $50 \mathrm{~mL}$ de amônio; completando-se com mais água destilada até ser atingido o volume final de $1000 \mathrm{~mL}$ (Figura 6). As membranas 
foram neutralizadas com a solução de hidróxido de amônio, não permanecendo em contato direto com esta solução, mas sim sob atmosfera da mesma, dentro de uma cúpula de vidro, por um período de 72 horas (Figura 7). Em seguida, as amostras foram colocadas para secar a temperatura ambiente $\left(23^{\circ} \mathrm{C}\right)$.
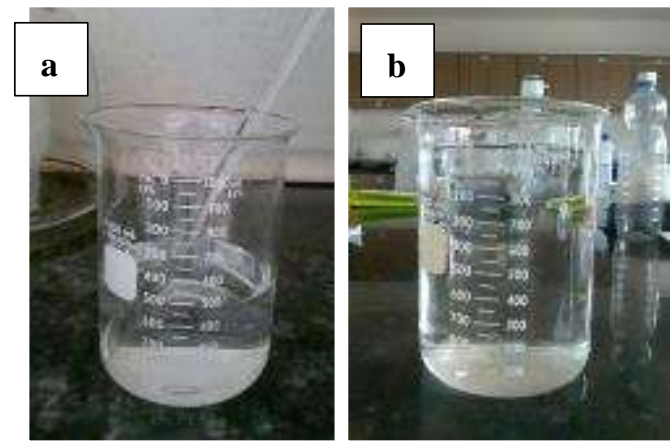

Figura 6: $500 \mathrm{~mL}$ de água destilada (a), Hidróxido de Amônio misturado à água destilada, com volume total de 1L (b).
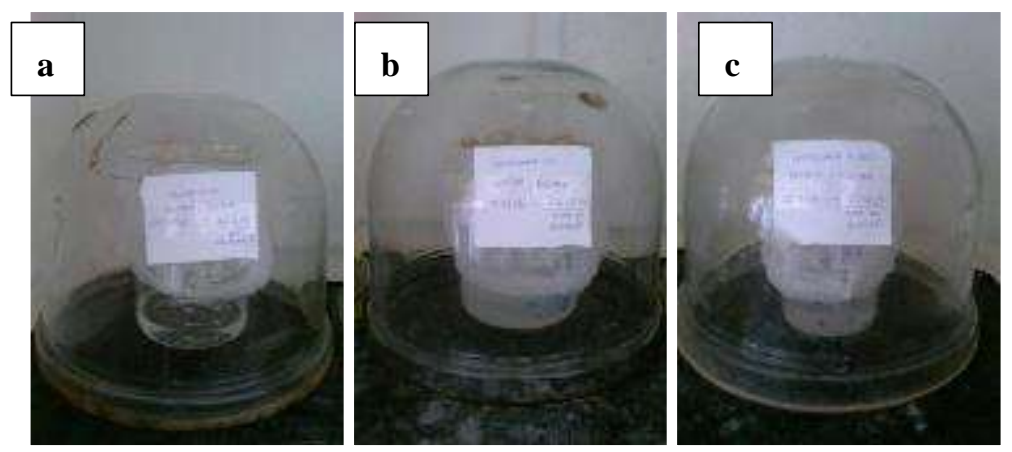

Figura 7: Processo de neutralização das membranas de quitosona pura (a), com 5\% de extrato vegetal (b) e com $20 \%$ de extrato vegetal (c) na capela de exaustão.

\subsection{Caracterização}

As membranas de quitosana desenvolvidas foram caracterizadas pelas técnicas de Microscopia Ótica (MO), Microscopia Eletrônica de Varredura (MEV), Teste de Resistência à Tração e Ensaio de Citotoxicidade. As análises foram realizadas no CERTBIO / UFCG.

\subsubsection{Microscopia Ótica (MO)}

A caracterização por MO, para avaliação morfológica das membranas, foi realizada em um Microscópio Óptico Digital Hirox Modelo KH-1300, de reflexão e transmissão, com canhão MX(G)2016Z e magnificação de 80x, acoplado a uma estação de Análise de Imagens, empregando-se o software 2D Measure.

\subsubsection{Microscopia Eletrônica de Varredura (MEV)}

A microscopia eletrônica de varredura analisa o material através da irradiação por um fino feixe de elétrons que interage com a superfície da amostra e origina uma série de radiações fornecendo, dessa forma, informação morfológica e topográfica sobre superfícies de sólidos, necessária para a compreensão do comportamento de superfícies [13].

A caracterização por MEV foi realizada no equipamento modelo Phenom Pro X, acoplado com sistema para microanálise química por Espectroscopia de Energia Dispersiva - EDS, sendo realizadas imagens usando magnificação de 260x, 500x e 1000x.

$\mathrm{Na}$ análise de $\mathrm{MEV}$, as membranas sem e com a incorporação da droga vegetal foram preparadas nas dimensões de $1 \mathrm{~cm}$ x $1 \mathrm{~cm}$.

\subsubsection{Teste de Resistência à Tração}

Esse tipo de análise é caracterizada pelo modo como as membranas respondem às solicitações mecânicas aplicadas, podendo estas ser do tipo de tensão ou deformação. A natureza desta resposta depende da estrutura química, temperatura, tempo e das condições de processamento do polímero. As membranas podem estar 
sujeitas a uma variedade de tensões durante o período de uso e sabe-se que as interações entre polímeros podem influenciar e modificar as propriedades de misturas em relação aos polímeros puros [14].

Nesse ensaio, as membranas foram inseridas no equipamento universal de ensaios mecânicos INSTRON 3366, empregando-se dois pratos de compressão (um fixo e um móvel), com uma média de carga de $10 \mathrm{KN}$, velocidade do travessão de $1,3 \mathrm{~mm} / \mathrm{min}$ e uma deformação do corpo de prova de $90 \%$.

\subsubsection{Ensaio de Citotoxicidade}

Esse ensaio objetiva avaliar o potencial quantitativo de citotoxicidade de um material, ou seja, a capacidade que um material tem em produzir efeitos letais ou subletais em sistemas biológicos a nível celular, devendo ser realizado com base na norma ISO 10993-5: 2009.

Para a realização do ensaio, as amostras de membranas foram cortadas em dimensões aproximadas de $5 \times 5 \mathrm{~mm}$. Todas as soluções, vidros, pipetas, e demais materiais empregados na análise estavam devidamente estéreis e todos os procedimentos foram executados sob condições assépticas e em ambiente estéril de uma câmara de fluxo laminar.

Para a obtenção do cálculo de incerteza foi empregada a seguinte sistemática: Teste de Grubbs para deteç̧ão de outliers; exclusão de outliers; cálculo de incerteza combinada e determinação das incertezas expandidas pelo produto entre as incertezas combinadas e um fator de abrangência $(\mathrm{k}=2)$.

\section{RESULTADOS E DISCUSSÕES}

As membranas obtidas foram denominadas de MQ (Membrana de quitosana pura), MQ5 (Membrana de quitosana com extrato vegetal a 5\%) e MQ20 (Membrana de quitosana com extrato vegetal a 20\%).

As membranas confeccionadas apresentaram aspecto liso, homogêneo e plano. Além disso, verificouse que as membranas de quitosana pura apresentaram-se de cor clara e homogêneas, confirmando os resultados obtidos por ROSENDO [11] e HOLANDA [15].

Somando-se a isso, as membranas com $5 \%$ de extrato vegetal apresentaram coloração esverdeada, e as membranas com $20 \%$ de extrato vegetal se apresentaram com uma coloração verde de tonalidade mais escura que as MQ5, demonstrando que, à medida que a concentração do extrato de Cissus verticillata (L.) Nicolson \& C.E. Jarvis foi incorporado, a coloração das membranas tornou-se mais esverdeada (Figura 8), corroborando com ROSENDO [11].

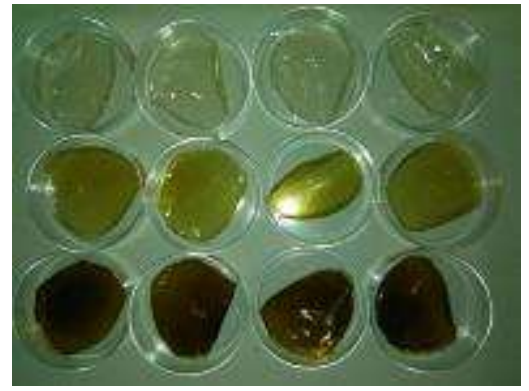

Figura 8: Aspecto macroscópico das membranas, observando-se as diferenças de coloração, à medida que a droga vegetal foi acrescentada.

\subsection{Resultados da Microscopia Ótica (MO)}

Na microscopia ótica das MQ (Figura 9), percebe-se uma superfície lisa, regular e sem a presença visível de poros.

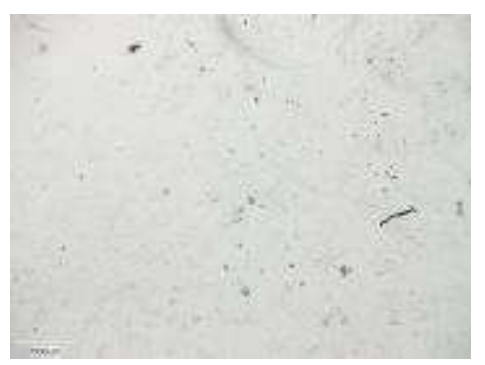

Figura 9: Microscopia ótica das MQ na magnificação de 80x, evidenciando a não presença de poros visíveis. 
Na Figura 10, constata-se a superfície das MQ5 e MQ20, as quais apresentaram pontos esverdeados, característicos da incorporação do EAB.

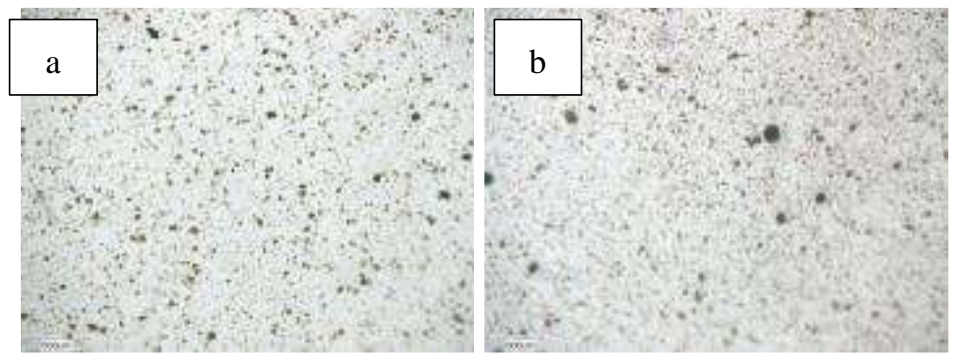

Figura 10: Microscopia ótica das MQ5 (a) e MQ20 (b) na magnificação de 80x.

\subsection{Resultados da Microscopia Eletrônica de Varredura (MEV)}

Na Figura 11, pode-se observar a MEV da MQ, com magnificação de 260x, 500x e 1000x, constatando-se na mesma um aspecto liso, plano, sem poros visíveis, corroborando com os estudos de LEMOS e LEMOS [10].
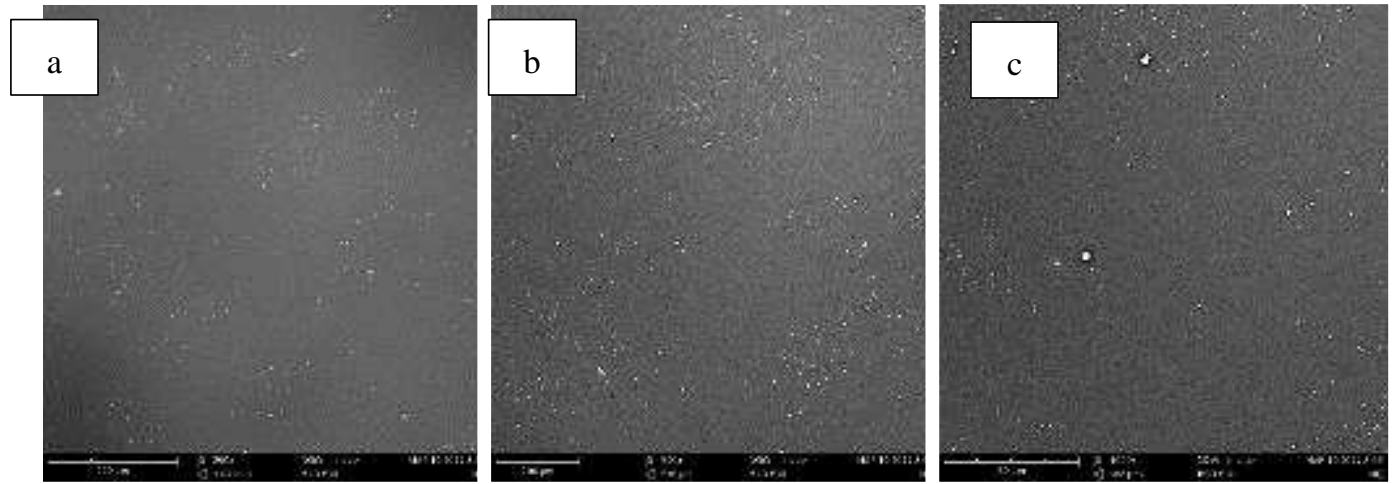

Figura 11: Imagens de MEV das MQ, com magnificação de 260x (a), 500x (b) e 1000x (c) respectivamente.

Já na MEV da amostra da MQ5, pode-se observar que teve uma alteração na morfologia das membranas em relação à MQ, sendo bem visível a presença de irregularidades (Figura 12).
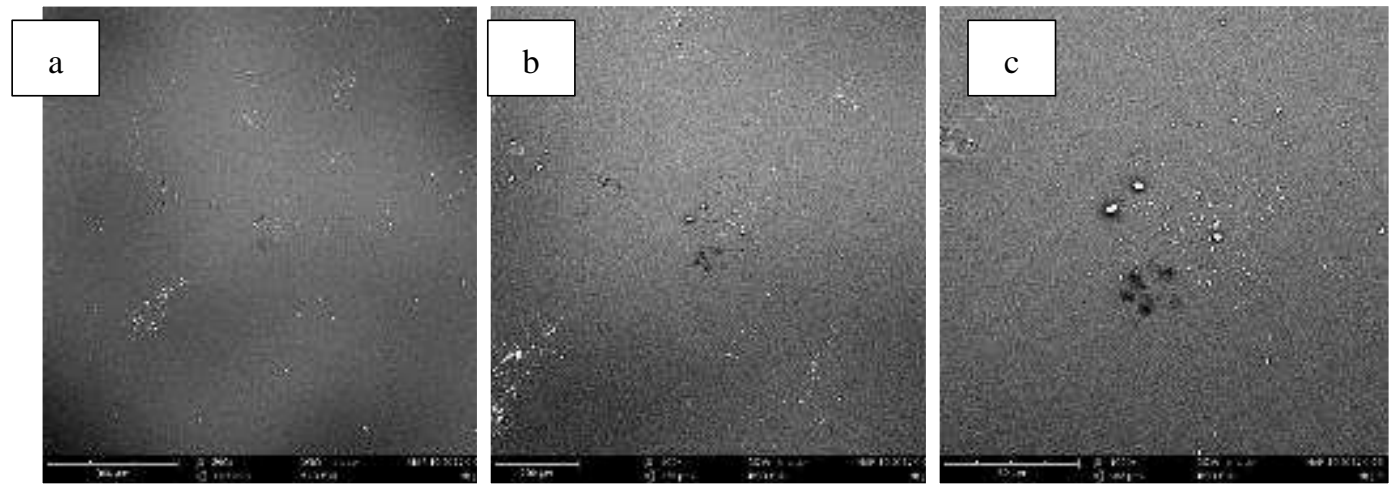

Figura 12: Imagens de MEV das membranas de quitosana com a incorporação do EAB a 5\% (MQ5), com magnificação de 260x, 500x e 1000x respectivamente.

$\mathrm{Na}$ análise de microscopia eletrônica de varredura das membranas de quitosana com extrato alcóolico bruto na concentração de $20 \%$ (MQ20), observa-se maiores quantidades de aglomerados e irregularidades em relação às MQ e MQ5 (Figura 13). 

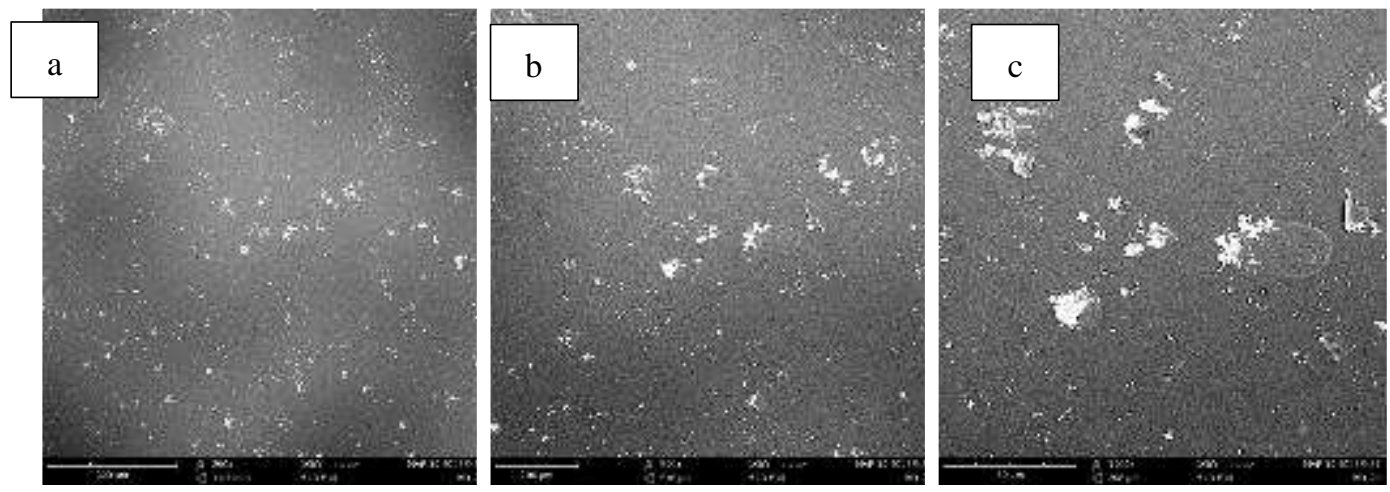

Figura 13: Imagens de MEV das MQ20, com magnificação de 260x, 500x e 1000x, evidenciando a grande quantidade de aglomerados e irregularidades.

Portanto, através da MEV, foram confirmados os resultados da Microscopia Ótica e pode-se notar que à medida que aumenta a concentração do extrato alcóolico bruto, aumenta a quantidade de irregularidades das membranas de quitosana.

\subsection{Resultados do Teste de Resistência à Tração}

Os ensaios mecânicos de resistência à tração foram realizados com o objetivo de avaliar o módulo elástico, deformação e tensão das membranas de quitosana com e sem extrato alcóolico bruto. Esse resultado é importante uma vez que demonstra o comportamento das membranas ao teste mecânico de resistência à tração.

Os resultados obtidos foram feitos através de três amostras de cada tipo de membrana de quitosana. Nas amostras de MQ, a deformação à tração ocorreu com uma média de 6,9\% com média de carga de 10,5 N e com um esforço à tração em resistência de tração máxima de 27,9 MPa (Figura 14).

Já as membranas com a adição de $5 \%$ do EAB, possuem uma carga de tensão máxima menor e uma deformação máxima similar à MQ. A média de carga de tensão foi 6,75 N, e com esforço à tração máxima de 17,115 MPa.

Todavia, as membranas com adição de $20 \%$ de extrato alcóolico bruto obtiveram uma média de carga de tração maior que MQ e MQ5, com 13,5 N e com um esforço à tração em resistência de tração máxima de 33,964 MPa.

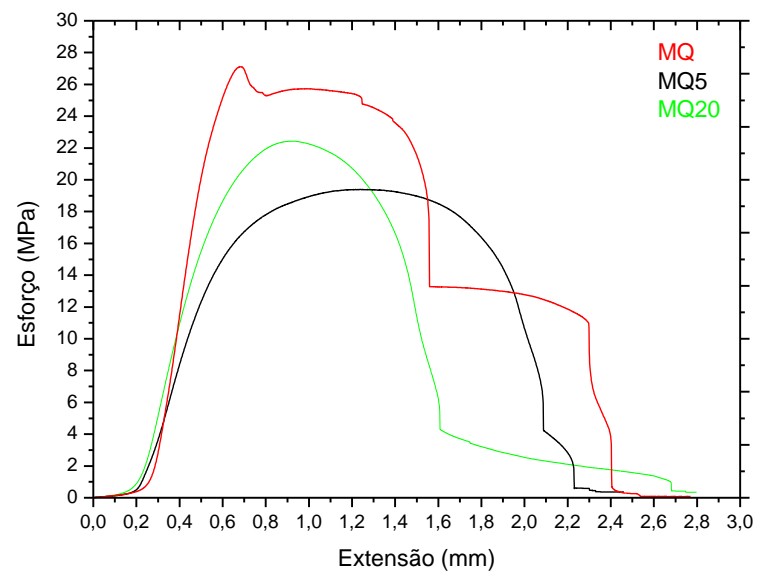

Figura 14: Esforço (MPa) e a Extensão (mm) máximas das amostras de MQ, MQ5 e MQ20.

Além disso, analisando a tensão e deformação das amostras das membranas de quitosana com e sem a adição de EAB (Tabela 1), pode-se observar que a MQ apresentou uma maior tensão e uma maior deformação máxima em relação a MQ5 e MQ20, porém o módulo de elasticidade foi maior em MQ20.

Tabela 1: Módulo de elasticidade, deformação máxima e tensão das médias das amostras das membranas de quitosana com e sem extrato alcoólico bruto. 


\begin{tabular}{l|l|l|l|}
\hline & $\begin{array}{l}\text { MÓDULO DE } \\
\text { ELASTICIDADE } \\
\text { (MPA/MM) }\end{array}$ & $\begin{array}{l}\text { DEFORMAÇÃO } \\
\text { MÁXIMA (\%) }\end{array}$ & $\begin{array}{l}\text { TENSÃO (MPA) / } \\
\text { DESVIO PADRÃO }\end{array}$ \\
\hline MQ & 8,594 & 6,943 & $20,884 \pm 8,61949$ \\
\hline MQ5 & 4,984 & 6,750 & $11,769 \pm 5,03570$ \\
\hline MQ20 & 10,351 & 5,442 & $19,698 \pm 5,98684$ \\
\hline
\end{tabular}

Pode-se concluir que a membrana de quitosana pura (MQ) possui uma maior resistência à tração longitudinal em relação às membranas com extrato vegetal.

\subsection{Resultados do Ensaio de Citotoxicidade}

Para as membranas serem usadas em sistemas biológicos, as mesmas devem ser, por segurança, não tóxicas e biocompatíveis. Os resultados do ensaio de citotoxicidade das MQ, MQ5 e MQ20 estão expressos na Tabela 2.

Tabela 2: Resultado em percentagem (\%) da viabilidade das MQ, MQ5 e MQ20 em sistemas biológicos a nível celular.

\begin{tabular}{l|l|}
\hline & $\%$ VIAB. \pm U (VIAB.) \\
\hline MQ & $80 \pm 7$ \\
\hline MQ5 & $77 \pm 6$ \\
\hline MQ20 & $76 \pm 5$ \\
\hline
\end{tabular}

Fazendo a comparação do percentual de citotoxicidade das membranas com o valor mínimo determinado por BISPO [16], que é de 50\%, e pela norma ISO 10993-5: 2009 que é de 70\%, subtraindo-se a incerteza, pode-se concluir que todas as membranas (MQ, MQ5 e MQ20) apresentaram-se viáveis para o uso em sistemas biológicos, uma vez que o percentual de viabilidade foi superior a 70\%. Esse resultado é importante uma vez que se pretende aplicar esse biomaterial como sistema de liberação de fármacos em pele/mucosa, com finalidade cicatrizante.

\section{CONCLUSÕES}

- Conclui-se que foi possível desenvolver membranas de quitosana com diferentes concentrações de Extrato Alcoólico Bruto na perspectiva da confecção de um sistema de liberação de fármacos com finalidade cicatrizante.

- Foi observado através da MO e MEV que as amostras apresentaram maior quantidade de irregularidades à medida que a droga vegetal foi incorporada.

- O grupo MQ apresentou uma maior resistência à tensão em relação aos demais grupos.

- Através do ensaio de citotoxicidade evidenciou-se que todas as membranas são capazes de interagir com os sistemas biológicos.

\section{AGRADECIMENTOS}

O presente trabalho foi realizado com apoio do CNPq, Conselho Nacional de Desenvolvimento Científico e Tecnológico - Brasil.

\section{BIBLIOGRAFIA}

[1] PIRES, A.L.R., BIERHALZ, A.C.K., MORAES, A.M., "Biomateriais: Tipos, aplicações e Mercado", Química Nova, São Paulo, v. 38, n. 7, pp. 957-971, Mai, 2015.

[2] KUNIYOSHI, J.N., “La Quitosana”, Revista de Química PUCP (Pontificia Universidad Católica del Perú), Lima, Peru, v. 26, n. 1-2, pp. 10-12, Ago, 2013.

[3] LARANJEIRA, M.C.M, FÁVERE, V.T., "Quitosana: Biopolímero Funcional com Potencial Industrial Biomédico”, Química Nova, Florianópolis, v. 32, n. 3, pp. 672-678, Mar/Abr, 2009. 
[4] TAVARIA, F.K., et al., "A quitosana como biomaterial odontológico: estado da arte”, Revista Brasileira de Engenharia Biomédica, Brasil, v. 29, n. 1, pp, 110-120, Mar, 2013.

[5] VEIGA, I.G., "Produção e caracterização de membranas de quitosana associada com outros biopolímeros para liberação controlada de anti-inflamatórios, Tese de D.Sc., FEQ/UNICAMP, Campinas, SP, Brasil, 2012.

[6] THEODORO, L.N., Análise da biocompatibilidade e ação antimicrobiana da matriz de hidrogel associada ao extrato de Stryphnodendron barbatiman. Dissertação de M.Sc., ICT/UNESP, São José dos Campos, SP, Brasil, 2017.

[7] BHUSAN, M., et al., "An analytical review of plants for anti-diabetic activity with their phytoconstituent \& mechanism of action", Internacional Journal of Pharmaceutical Sciences and Research, v. 1, pp. 29-45, 2010 .

[8] RIZVI, S., MISHRA, N., "Traditional Indian Medicines Used for the Management of Diabetes Mellitus", Journal of Diabetes Research, v. 2013, pp. 1-11, 2013.

[9] FIRMINO, F.C., BINSFELD, P.C., A biodiversidade brasileira como fonte de medicamentos para o SUS. Goiás, PUC, http://www.cpgls.pucgoias.edu.br. Acessado em janeiro de 2017.

[10] LEMOS, H.P.J, LEMOS, A.L.A., "Efetividade e segurança do vegetal Cissus sicyoides ("insulina vegetal”) como fitoterápico hipoglicemiante”, Revista Diagnóstico \& Tratamento, Santos, v. 19, pp. 129-131, Jul/Set, 2014.

[11] ROSENDO, R.A., Desenvolvimento e Caracterização de Scaffolds de Quitosana/ Cissus verticillata (L.). Tese de D.Sc., PPGCEMat./UFCG, Campina Grande, PB, Brasil, 2016.

[12] ROSENDO, R.A., Avaliação da eficácia da Cissus verticillata (L.), em portadores de diabetes melito tipo 2, através da concentração de glicose na saliva e no sangue, Dissertação de M.Sc., PPGO/UFPB, João Pessoa, PB, Brasil, 2009.

[13] FOOK, M.V.L., Desenvolvimento da técnica de deposição de hidroxiapatita na superfície de polietileno de ultra-peso molecular para aplicação como biomaterial, Tese de D.Sc., IQ/UNESP, Araraquara, SP, Brasil, 2005.

[14] PINHEIRO, G.K., Desenvolvimento de "scaffolds" a partir de compósitos contendo blendas poliméricas à base de quitosana para reconstituição tecidual, Relatório apresentado ao Curso de Graduação em Química da Universidade Federal de Santa Catarina, Santa Catarina, 2008.

[15] HOLANDA, A.L., Membranas de quitosana para uso em sistema de liberação controlada de insulina: síntese e caracterização, Tese de D.Sc., PPGCEMat./UFCG, Campina Grande, PB, Brasil, 2011.

[16] BISPO, V.M., Estudo do efeito da reticulação por Genipin em suportes biocompatíveis de Quitosana$P V A$, Tese de D.Sc., UFMG, Belo Horizonte, MG, Brasil, 2009.

\section{ORCID}

Antônio Alberto Souza Neto

Luanna Abílio Diniz Melquíades de Medeiros

Marcus Vinícius Lia Fook

Rafael Rodrigues de Siqueira

Rossemberg Cardoso Barbosa

Walter Gleybson Antas de Moraes

Rosana Araújo Rosendo https://orcid.org/0000-0003-3116-1336

https://orcid.org/0000-0002-1630-3968

https://orcid.org/0000-0002-8566-920x

https://orcid.org/0000-0002-0748-3506

https://orcid.org/0000-0002-8551-5251

https://orcid.org/0000-0002-2128-5573

https://orcid.org/0000-0002-3795-8832 